\title{
POLYNOMIAL BASES FOR COMPACT SETS IN THE PLANE
}

\author{
BY \\ VICTOR MANJARREZ
}

1. Introduction. This work is essentially a generalization, in two directions, of theorems about the Taylor series expansion of functions analytic on the closed unit disk in the plane. In the first place, using work of J. L. Walsh, we replace the closed unit disk by any compact set $E$ whose complement $E^{\prime}$ is connected, and regular in the sense that $E^{\prime}$ has a Green's function with pole at infinity. Such a set $E$ will be called a coregular set. In the second place, as a generalization of work of B. Cannon and J. M. Whittaker, the Taylor series expansion is replaced by a large class of expansions of the form:

$$
f(z)=\sum_{0}^{\infty} A_{k} P_{k}(z)
$$

where $\left(P_{k}\right)$ is a simple polynomial basis (that is, each $P_{k}$ is a polynomial of exact degree $k$ ) which depends on $E$ but not on $f$, each $A_{k}$ is a constant depending on $f$, and the convergence is uniform on each compact subset of some open set $O$ containing $E$. Such convergence will be called compact-open on $E$ or compact-open in $O$.

The polynomial bases we study will be shown to give rise to maximal convergence as defined by Walsh. We shall state and prove, for such bases, exact analogs of Ostrowski's theorems concerning the relation between overconvergence of the Taylor series and lacunary structure of the Taylor series. Finally, the class of bases for which our results hold will be shown to include certain classical polynomial bases, such as the Faber polynomials and all kinds of orthogonal polynomials.

This work represents part of a thesis done under the direction of Professor J. L. Walsh. It is a pleasure to acknowledge his interest, encouragement, and help, which were invaluable throughout the thesis work.

2. Some notation. Let $E$ be a coregular set and let $F$ be the Green's function with pole at infinity for the complement $E^{\prime}$ of $E$. Let $K=\lim _{z \rightarrow \infty}|z| \exp (-F(z))$. $K$ is called the capacity or transfinite diameter of $E$. We shall assume $K>0$. For each extended real number $x \geqq 1$ let $E_{x}$ be the set of all $z$ such that $z$ is in $E$ or $\exp (F(z))<x$, and let $L_{x}$ be the boundary of $E_{x}$.

Let $\left(Z_{n}\right)$ be a sequence of points of $E$ such that, if $f$ is any function analytic on $E$ and $S_{n}$ is the interpolation polynomial for $f$ in the points $Z_{1}, \ldots, Z_{n+1}$, then the sequence $\left(S_{n}\right)$ converges to $f$ uniformly on $E$. $S_{n}$ is called the $n$th interpolation

Presented to the Society, December 27, 1966; received by the editors April 4, 1967. 
polynomial for $f$ in the points $\left(Z_{n}\right)$. Let $Q_{0}(z)=1, Q_{n}(z)=\left(z-Z_{1}\right) \cdots\left(z-Z_{n}\right)$. We shall call $\left(Q_{n}\right)$ the interpolation basis for $E$ in the points $\left(Z_{n}\right)$.

The largest positive extended real number $X$ such that $f$ is analytic in $E_{X}$ will be called the modulus of analyticity of $f$ with respect to $E$. Note that $S_{n}(z)=\sum_{0}^{n} B_{k} Q_{k}(z)$ where

$$
B_{k}=(1 / 2 \pi i) \int_{L_{y}}\left(f(z) / Q_{k+1}(z)\right) d z
$$

for any $y$ such that $1<y<X$. Thus $f(z)=\sum_{0}^{\infty} B_{n} Q_{n}$, where the convergence is compact-open in $E_{X}$, and

$$
\lim \sup \left|B_{n}\right|^{1 / n}=1 / K X .
$$

$\sum_{0}^{\infty} B_{n} Q_{n}$ is called the interpolation series for $f$ in the points $\left(Z_{n}\right)$. See Walsh $[9$, pp. $65,74,170,159,157,52-54]$ and $[8$, p. 606] for the above definitions and assertions.

Let $B=\left(B_{n}\right)$ and $Q=\left(Q_{n}\right)$ be sequences, and let $D=\left[D_{n k}\right]$ and $G=\left[G_{n k}\right]$ be infinite matrices. $D \cdot G$ will denote the matrix product of $D$ and $G$ whenever all the series thus defined converge. $B \cdot G, G \cdot Q$ and $B \cdot Q$ are defined by considering $B$ as a matrix with one row and $Q$ as a matrix with one column. $|B|$ will denote the sequence $\left(\left|B_{n}\right|\right)$ and $|D|$ will denote the matrix $\left[\left|D_{n k}\right|\right]$, while $N(B)$ will denote lim sup $\left|B_{n}\right|^{1 / n}$. We will sometimes write $N\left(B_{n}\right)$ for $N(B)$.

3. Effective polynomial bases. For the remainder of the paper let $Q=\left(Q_{n}\right)$ be an interpolation basis belonging to the coregular set $E$, with capacity $K>0$. Let $P=\left(P_{n}\right)$ be any simple polynomial basis, and let $G=\left[G_{n k}\right]$ be the (unique) matrix of complex numbers such that

$$
Q_{n}=\sum_{k=0}^{\infty} G_{n k} P_{k} \quad(n=0,1,2, \ldots)
$$

Note that (3.1) can be written $Q=G \cdot P$, and that $G$ is lower triangular. For each $x \geqq 1$ let $M(x)=\left(M_{n}(x)\right)$, where

$$
M_{n}(x)=\left(\max \left|P_{n}(z)\right|, z \text { on } L_{x}\right) \quad(n=0,1,2, \ldots) .
$$

Let $R(x)=\left(R_{n}(x)\right)$ be defined by

$$
R_{n}(x)=\sum_{k=0}^{\infty}\left|G_{n k}\right| M_{k}(x) \quad(n=0,1,2, \ldots),
$$

and define $I(x)=\lim \sup \left(R_{n}(x)\right)^{1 / n}$. Note that $R(x)=|G| \cdot M(x)$ and $I(x)=N(R(x))$,

By the maximum principle, $I(x) \leqq I(y)$ when $x \leqq y$. Moreover, $\left|Q_{n}(z)\right| \leqq R_{n}(x)$ for $z$ on $L_{x}$, and since

$$
\lim \left|Q_{n}(z)\right|^{1 / n}=K \exp (F(z))
$$

where the convergence is compact-open in $E^{\prime}[9$, p. 159], we have $I(x) \geqq K x$ for 
$x>1$. The fact that $I(1) \geqq K$ follows from the lemma in [9, p. 77], applied to the $\left(Q_{n}(z)\right)$ on $L_{1}$.

LEMma. $I(x)$ is independent of $Q$.

Proof. Let $q=\left(q_{n}\right)$ be any other interpolation basis for $E$. Let $g=\left[g_{n k}\right]$ be the matrix such that $q=g \cdot P$, and let $r(x)=|g| \cdot M(x)$. We wish to show that $N(r(x))$ $=N(R(x))$. Let $D$ be the matrix such that $Q=D \cdot q$. Note that $D$ is lower triangular and $D_{n n}=1$ for all $n$. Also, since $D$ and $g$ are lower triangular and $P$ is a basis, we have $G \cdot P=Q=D \cdot q=D \cdot(g \cdot P)=(D \cdot g) \cdot P$, so $G=D \cdot g$.

We consider first the case $K=1$. Let $y$ be any number greater than 1. From (2.1) we get

so that

$$
D_{n k}=(1 / 2 \pi i) \int_{L_{y}}\left(Q_{n}(z) / q_{k+1}(z)\right) d z
$$

$$
\left|D_{n k}\right| \leqq c\left(\max \left|Q_{n}(z)\right|, z \text { on } L_{y}\right) /\left(\min \left|q_{k+1}(z)\right|, z \text { on } L_{y}\right)
$$

where $c$ is a positive constant. Applying (3.4) with $K=1$ to $Q$ and $q$, we get

and

$$
\lim _{n} \inf \left(\min \left(\min \left|q_{k+1}(z)\right|, z \text { on } L_{y}\right), k=0,1, \ldots, n\right)^{1 / n} \geqq 1
$$

$$
\lim _{n} \sup \left(\max \left|D_{n k}\right|, k=0,1, \ldots, n\right)^{1 / n} \leqq y .
$$

Since this is so for all $y>1$, and since $\left|D_{n n}\right|=1$ for all $n$, we get

$$
\lim _{n} \sup \left(\max \left|D_{n k}\right|, k=0,1, \ldots, n\right)^{1 / n}=1 .
$$

Now $N(R(x))=N(|G| \cdot M(x))=N(|D \cdot g| \cdot M(x)) \leqq N(|D| \cdot|g| \cdot M(x))=N(|D| \cdot r(x))$. But $N(|D| \cdot r(x)) \leqq N(r(x))$ because of (3.5) and the fact that $N(r(x)) \geqq K x=x \geqq 1$. Reversing the roles of $r$ and $R$ we get $N(r(x)) \leqq N(R(x))$ and so $N(r(x))=N(R(x))$. This completes the proof of the lemma for the case $K=1$. The proof for the general case follows from a consideration of the polynomial basis $\left(P_{n}(K z)\right)$, on the set $(1 / K) E=$ the set of all $z$ such that $K z$ is in $E$, since $(1 / K) E$ has capacity 1 . The corresponding interpolation bases are $\left(Q_{n}(K z) / K^{n}\right)$ and $\left(q_{n}(K z) / K^{n}\right)$. This completes the proof of the lemma.

Definition. $P$ is called effective on $\mathrm{Cl}\left(E_{x}\right)=$ the closure of $E_{x}$ if and only if, for some interpolation basis $Q$, any function $f$ analytic on $\mathrm{Cl}\left(E_{x}\right)$ can be expanded in the form (1.1), the convergence being uniform on $\mathrm{Cl}\left(E_{x}\right)$, with $A=\left(A_{k}\right)$ defined by

$$
A_{k}=\sum_{n=0}^{\infty} B_{n} G_{n k} \quad(k=0,1,2, \ldots)
$$

where $B$ is defined by (2.1) for some $y>1$, and $G$ is defined by (3.1). Note that (3.6) can be written $A=B \cdot G$.

THEOREM 1. $P$ is effective on $\mathrm{Cl}\left(E_{x}\right)$ if and only if $I(x)=K x$. 
Proof. Let $I(x)=K x$ for some $x \geqq 1$ and let $f$ be analytic on $\mathrm{Cl}\left(E_{x}\right)$. Let $f=B \cdot Q$ for some interpolation basis $Q$, define $G$ by (3.1), and define $A$ by (3.6). From (2.2) it follows that $N(B)<1 / K x$, while $N(R(x))=K x$, so $|B| \cdot R(x)$ converges absolutely. Thus $f=B \cdot Q=B \cdot(G \cdot P)=(B \cdot G) \cdot P=A \cdot P$, where the absolute convergence of $|B| \cdot R(x)$ implies the convergence of every term in $B \cdot G$ and the uniform convergence of $A \cdot P$ on $\mathrm{Cl}\left(E_{x}\right)$, as well as the equality $B \cdot(G \cdot P)=(B \cdot G) \cdot P$.

Conversely, let $I(x) \neq K x$ and let $X$ be any number such that $K x<K X<I(x)$. We construct a function $f$, with modulus of analyticity $X$ with respect to $E$, such that $f$ cannot be expanded in the necessary way. Let $C_{k}=\left(G_{n k}\right)$ be the $k$ th column of $G$, and let $B=\left(B_{n}\right)=\left(1 /(K X)^{n}\right)$.

Case 1. Suppose that for some $k, B \cdot\left|C_{k}\right|$ does not converge. Choose real numbers $\left(y_{n}\right)$ such that $G_{n k}=\left|G_{n k}\right| \exp \left(i y_{n}\right)$ and let $b=\left(b_{n}\right)=\left(\exp \left(-i y_{n}\right) /(K X)^{n}\right)$. Define $f=b \cdot Q$. Then from (2.2) $f$ has modulus of analyticity $X$, but $f$ cannot be expanded in the necessary way, since if it could, then $A_{k}=b \cdot C_{k}=B \cdot\left|C_{k}\right|$, which is a contradiction.

Case 2. Suppose $B \cdot\left|C_{k}\right|$ converges for all $k$. If $M$ is defined by (3.2) then

$$
K X<I(x) \leqq \lim _{n} \sup \left(\max \left|G_{n k}\right| M_{k}(x), k=0,1, \ldots, n\right)^{1 / n}
$$

and so there exist sequences $n(j)$ and $k(j)$ of positive integers, with $n(j)$ strictly increasing, such that

$$
\left|G_{n(j) k(j)}\right| M_{k(j)}(x)>(K X)^{n(j)} .
$$

Now the terms $G_{n(j) k(j)}$ cannot all belong to a finite number of the $C_{k}$, since if infinitely many belong to $C_{k}$ for some $k$, then $B \cdot\left|C_{k}\right|$ would not converge. Consequently we may assume that $k(j)$ is also strictly increasing. Choose three sequences $u(j), v(j)$ and $w(j)$ of positive integers in the following way: let $u(1)=n(1)$, $v(1)=k(1)$. Choose $w(1)>u(1)$ such that

$$
\left|\sum_{h=w(1)}^{\infty} G_{h v(1)} /(K X)^{h}\right|<(1 / 2)\left|G_{u(1) v(1)}\right| /(K X)^{u(1)} .
$$

If $u(j), v(j)$ and $w(j)$ have been chosen, choose $u(j+1)$ and $v(j+1)$ such that $u(j+1)=n(r), v(j+1)=k(r)$ for some $r, u(j+1)>w(j)$, and $G_{n v(j+1)}=0$ for $n<w(j)$. This last requirement can be met by taking $v(j+1) \geqq w(j)$. Finally, choose $w(j+1)>u(j+1)$ such that

$$
\left|\sum_{h=w(j+1)}^{\infty} G_{h v(j+1)} /(K X)^{h}\right|<(1 / 2)\left|G_{u(j+1) v(j+1)}\right| /(K X)^{u(j+1)} .
$$

Let $b=\left(b_{n}\right)$ where $b_{n}=1 /(K X)^{n}$ when $n=u(j)$ for some $j$, and $b_{n}=0$ otherwise. Define $f=b \cdot Q$. From (2.2) $f$ has modulus of analyticity $X$. Now for all $j$,

Thus

$$
\begin{aligned}
\left|A_{v(j)}\right| & =\left|b \cdot C_{v(j)}\right|=\left|G_{u(j) v(j)} /(K X)^{u(j)}+\sum_{h=w(j)}^{\infty} b_{h} G_{h v(j)}\right| \\
& >(1 / 2)\left|G_{u(j) v(j)}\right| /(K X)^{u(j)} .
\end{aligned}
$$

$$
\left|A_{v(j)}\right| M_{v(j)}(x)>(1 / 2)\left|G_{u(j) v(j)}\right| M_{v(j)}(x) /(K X)^{u(j)}>1 / 2
$$


by (3.7). Thus $A \cdot P$ does not converge uniformly on $L_{x}$. This completes the proof of the theorem.

Because of Theorem 1, the function $I$, defined for all $x \geqq 1$, is called the index of effectivity of $P$ on $E$. By [9, p. 77], $M_{n}(y) \leqq(y / x)^{n} M_{n}(x)$ whenever $x<y$, and so $I(y) \leqq(y / x) I(x)$. Together with the fact that $I(x) \leqq I(y)$ whenever $x<y$, we conclude that either $I(x)=\infty$ for all $x \geqq 1$ or else $I$ is finite-valued and continuous for all $x \geqq 1$. Moreover, suppose $P$ is effective on $\mathrm{Cl}\left(E_{x}\right)$ for some $x$ and let $y$ be any number $>x$. Then $I(y) \leqq(y / x) I(x)=(y / x) K x=K y$, and since $I(y) \geqq K y$, we get $I(y)=K y$, so $P$ is effective on $\mathrm{Cl}\left(E_{y}\right)$.

Because of the lemma, we know that the effectivity of $P$ does not depend on the interpolation basis $Q$. The coefficients $A$ in (1.1) also do not depend on $Q$ when $P$ is effective, a consequence of

THEOREM 2. Let $P$ be a simple polynomial basis, and let $f(z)=\sum_{0}^{\infty} A_{n} P_{n}(z)$, where the convergence is absolute and compact-open on an open set containing $\mathrm{Cl}\left(E_{x}\right)$ for some $x \geqq 1$. Let $G$ be defined by (3.1) for any interpolation basis $Q$, and let $C_{j}$ be the $j$ th column of $G$. If $N\left(C_{j}\right) \leqq K x$ for some $j$, then $A_{j}=B \cdot C_{j}$, where $B$ is defined by (2.1).

Proof. Let $A=\left(A_{n}\right)$, and let $y$ be any number $>x$ such that $A \cdot P$ converges absolutely and uniformly on $L_{y}$. From $(2.2), N(B) \leqq 1 / K y<1 / K x$, while $N\left(C_{j}\right) \leqq K x$ so $B \cdot C_{j}$ converges. Let $D=\left[D_{n k}\right]$ be the matrix inverse of $G(G$ is lower triangular with nonzero diagonal elements). Then $P=D \cdot Q$ since $D$ and $G$ are lower triangular, and from (2.1) we conclude that $D$ is given by:

$$
D_{n k}=(1 / 2 \pi i) \int_{L_{y}}\left(P_{n}(z) / Q_{k+1}(z)\right) d z
$$

Again from (2.1) we get for each $k$ :

$$
\begin{aligned}
B_{k} & =(1 / 2 \pi i) \int_{L_{y}}\left(f(z) / Q_{k+1}(z)\right) d z \\
& =\sum_{n=0}^{\infty} A_{n}(1 / 2 \pi i) \int_{L_{y}}\left(P_{n}(z) / Q_{k+1}(z)\right) d z=\sum_{n=0}^{\infty} A_{n} D_{n k} .
\end{aligned}
$$

Thus $B=A \cdot D$. Since $A \cdot P$ converges absolutely and uniformly on $L_{y}$, the function $H$ defined by: $H(z)=\sum_{n=0}^{\infty}\left|A_{n} P_{n}(z)\right|$ is continuous on $L_{y}$. Therefore from (3.8), the $k$ th term of $|A| \cdot|D|$ satisfies the following:

$$
\begin{aligned}
\sum_{n=0}^{\infty}\left|A_{n}\right|\left|D_{n k}\right| & \leqq 1 /(2 \pi) \sum_{n=0}^{\infty}\left|A_{n}\right| \int_{L_{y}}\left|P_{n}(z) / Q_{k+1}(z)\right||d z| \\
& =1 /(2 \pi) \int_{L_{y}}\left(H(z) /\left|Q_{k+1}(z)\right|\right)|d z| \leqq J /\left(\min \left|Q_{k+1}(z)\right|, z \text { on } L_{y}\right),
\end{aligned}
$$

where $J$ is a positive constant. From (3.4) we conclude that $N(|A| \cdot|D|) \leqq 1 / K y$, so $(|A| \cdot|D|) \cdot\left|C_{j}\right|$ converges. Therefore, $B \cdot C_{j}=(A \cdot D) \cdot C_{j}=A \cdot\left(D \cdot C_{j}\right)=A_{j}$. This completes the proof of the theorem. 
Corollary. Let $P$ be effective on $\mathrm{Cl}\left(E_{x}\right)$ for some $x \geqq 1$ and let $f$ be analytic on $\mathrm{Cl}\left(E_{x}\right)$. Then the coefficients $A$ in (1.1) are unique.

Proof. Let $f=B \cdot Q$ for any interpolation basis $Q$, and let $X$ be the modulus of analyticity of $f$. From (2.2), $N(B)=1 / K X$, and so, if $R(x)$ is defined by (3.3), then from the first part of the proof of Theorem 1 we see that $|B| \cdot R(y)$ converges for all $y$ such that $N(R(y))<K X$. But from the remarks following Theorem $1, N(R(y))=K y$ for all $y \geqq x$, so the convergence of $|B| \cdot R(y)$ is compact-open on $1 \leqq y<X$. Thus the convergence of $A \cdot P$ is absolute and compact-open in $E_{x}$. If $G$ is defined by (3.1) then $\left|G_{n j}\right| \leqq R_{n}(x) / M_{j}$, so that if $C_{j}$ is the $j$ th column of $G$ then $N\left(C_{j}\right) \leqq N(R(x))$ $=I(x)=K x$. This completes the proof of the corollary.

See Whittaker [10, pp. 5-12, 18-20, 60-62] for the definitions and results in this section (except the lemma) for the special case $E=$ the closed unit disk, $Q_{n}(z)=z^{n}$ for all $n$. Whittaker also treats effectivity in $E_{x}$ for this special case, and many of those results can also be generalized.

4. Maximal convergence. Suppose $f$ is analytic on $E$ with modulus of analyticity $X$. For each $n$ let $S_{n}$ be a polynomial of degree $n$, and let $m=\left(m_{n}\right)$ where $m_{n}$ $=\left(\max \left|f(z)-S_{n}(z)\right|, z\right.$ on $\left.E\right)$. If $N(m) \leqq 1 / X$ then $\left(S_{n}\right)$ is said to converge maximally to $f$ on $E[9$, pp. 79,80$]$.

THEOREM 3. If $P$ is effective on $E$ then $P$ always gives rise to maximal convergence on $E$.

Proof. Let $f$ be analytic on $E$ with modulus of analyticity $X$. Then $f=A \cdot P$, where $A=B \cdot G, B$ being defined by (2.1), and $G$ by (3.1) for any interpolation basis $Q$. Now if $x$ is any number such that $1 \leqq x<X$ then, from the first part of the proof of Theorem $1, A \cdot P=(B \cdot G) \cdot P=B \cdot(G \cdot P)=B \cdot Q$ on $L_{x}$ and therefore on $E$. If $M(x)$ and $R(x)$ are defined by (3.2) and (3.3), then for $z$ on $L_{x}$ we have:

$$
\begin{aligned}
\left|f(z)-\sum_{k=0}^{n} A_{k} P_{k}(z)\right| & =\left|\sum_{k=n+1}^{\infty} A_{k} P_{k}(z)\right| \leqq \sum_{k=n+1}^{\infty}\left|A_{k}\right|\left|P_{k}(z)\right| \\
& \leqq \sum_{k=n+1}^{\infty}\left(\sum_{j=k}^{\infty}\left|B_{j}\right|\left|G_{j k}\right|\right) M_{k}(x) \leqq \sum_{j=n+1}^{\infty}\left|B_{j}\right| R_{j}(x)
\end{aligned}
$$

since $G$ is lower triangular. From (2.2) and (3.4) we get:

$$
\lim _{.} \sup \left(\sum_{j=n+1}^{\infty}\left|B_{j}\right| R_{j}(x)\right)^{1 / n} \leqq x / X .
$$

Letting $x=1$ completes the proof of the theorem.

It is not true that every simple polynomial basis which gives rise to maximal convergence is effective. For example, let $E$ be the closed unit disk, which has capacity $K=1$. Let $Q_{n}(z)=z^{n}$ for all $n$. Let $x$ be any number $>1$. Let $P_{0}(z)=1$, $P_{n}(z)=(z-x) z^{n-1}$ for all $n \geqq 1$. If $G$ is defined by (3.1) then $G_{n k}=x^{n-k}$, so 
$I(1) \geqq x>1$, and by Theorem $1, P$ is not effective on $E$. On the other hand, any function analytic on $E$ can be expanded in the form (1.1), with maximal convergence [9, p. 159].

The effectivity of a polynomial basis $P$ on $\mathrm{Cl}\left(E_{x}\right)$ for some coregular set $E$ and some $x \geqq 1$ does not imply anything about the maximum values of $\left|P_{n}(z)\right|$ on $L_{x}$. For, suppose $P$ is any polynomial basis and let $\left(T_{k}\right)$ be any sequence of nonzero complex numbers. Let $p=\left(p_{k}\right)=\left(T_{k} P_{k}\right)$ and let $g=\left[g_{n k}\right]$ be defined by $Q=g \cdot p$, where $Q$ is any interpolation basis. For any $x \geqq 1$ let $M(x)$ and $R(x)$ be defined by (3.2) and (3.3), let $m(x)=\left(m_{n}(x)\right)$ be defined by

$$
m_{n}(x)=\left(\max \left|p_{n}(z)\right|, z \text { on } L_{x}\right)=\left|T_{n}\right|\left(\max \left|P_{n}(z)\right|, z \text { on } L_{x}\right)=\left|T_{n}\right| M_{n}(x),
$$

and let $r(x)=|g| \cdot m(x)$. Now if $Q=G \cdot P$ then $g_{n k}=G_{n k} / T_{k}$ and so $r(x)=|g| \cdot m(x)$ $=|G| \cdot M(x)=R(x)$. Thus the values of the index of effectivity are unchanged if the $P_{n}$ are multiplied by nonzero constants, and so, from Theorem 1, effectivity is unaffected.

We may therefore assume, when it is convenient, that $P$ is made up of monic polynomials. On the other hand, with this assumption we have $N(M(x))=K x$. In fact, we even have:

THEOREM 4. Let $P$ be effective on $\mathrm{Cl}\left(E_{x}\right)$ for some $x \geqq 1$, and suppose every $P_{n}$ is monic. Then $\lim \left(M_{n}(x)\right)^{1 / n}=K x$.

Proof. Let $D$ be defined by $P=D \cdot Q$ for any interpolation basis $Q$. Then $D$ is given by (3.8) for any $y>1$. Moreover, $D_{n n}=1$ for all $n$, since $P_{n}$ and $Q_{n}$ are monic. Let $\left(M_{k(n)}(y)\right)$ be any subsequence of $M(y)$. Then from (3.8):

$$
1 \leqq c\left(\max \left|P_{k(n)}(z)\right|, z \text { on } L_{y}\right) /\left(\min \left|Q_{k(n)+1}(z)\right|, z \text { on } L_{y}\right)
$$

where $c$ is a positive constant. From (3.4) we get $K y \leqq \lim \sup _{n}\left(M_{k(n)}(y)\right)^{1 / k(n)}$. On the other hand, since $D_{n n}=1$ for all $n, M_{k(n)}(y) \leqq R_{k(n)}(y)$, so

$$
K y \leqq \lim _{n} \sup \left(M_{k(n)}(y)\right)^{1 / k(n)} \leqq I(y),
$$

for all $y>1$. Now if $x>1$ then (3.9) and Theorem 1 show that

$$
\lim \sup _{n}\left(M_{k(n)}(x)\right)^{1 / k(n)}=K x
$$

for every subsequence of $M(x)$. Therefore the theorem is proved for $x>1$. If $x=1$ then (3.9) shows that $\lim \sup _{n}\left(M_{k(n)}(1)\right)^{1 / k(n)} \leqq K y$ for all $y>1$ so

$$
\lim _{n} \sup _{n}\left(M_{k(n)}(1)\right)^{1 / k(n)} \leqq K,
$$

while $\left[9\right.$, p. 77] and our theorem for $x>1$ show that $\lim \sup _{n}\left(M_{k(n)}(1)\right)^{1 / k(n)} \geqq K$, so the theorem is proved for $x=1$. This completes the proof of the theorem.

Corollary. Let $P$ be effective on $E$ and suppose every $P_{n}$ is monic. Let $f$ be analytic on $E$, with modulus of analyticity $X$. If $A=\left(A_{n}\right)$ is defined by (1.1) then 
$N(A)=1 / K X$. Moreover, if $O$ is any bounded continuum, not a single point, in $E^{\prime}$, then

$$
\lim \left(\max \left|P_{n}(z)\right|, z \text { in } O\right)^{1 / n}=K \max (\exp (F(z)), z \text { in } O) .
$$

Proof. Since $A \cdot P$ converges maximally on $E, N\left(A_{n} M_{n}(X)\right)=1[8$, p. 606]. Thus $N(A)=1 / K X$. Now let $x=(\max \exp (F(z)), z$ in $O)$, and let $m_{n}=\left(\max \left|P_{n}(z)\right|, z\right.$ in $\left.O\right)$. From the maximum principle and the theorem, $N\left(m_{n}\right) \leqq K x$. Suppose there exists a subsequence $\left(m_{k(n)}\right)$ such that $\lim _{n}\left(m_{k(n)}\right)^{1 / k(n)}<K x$. Let $a_{j}=1 /(K x)^{j}$ when $j=k(n)$, $a_{j}=0$ otherwise. From the theorem, $N\left(a_{j} M_{j}(y)\right)=y / x$ for all $y \geqq 1$, so $a \cdot P$ converges maximally to a function with modulus of analyticity $x$. But $N\left(a_{j} m_{j}\right)<1$, which contradicts $[8$, p. 606]. This completes the proof of the corollary.

5. Overconvergence. Suppose $f$ is analytic on $E$ with modulus of analyticity $X$. Let $P$ be any simple polynomial basis effective on $E$, and let $S_{n}=\sum_{j=0}^{n} A_{j} P_{j}$, where $A=\left(A_{j}\right)$ is defined by (1.1). From the first part of the proof of Theorem $1,\left(S_{n}\right)$ converges uniformly on any compact subset of $E_{X}$. Now $\left(S_{n}\right)$ cannot converge uniformly on any neighborhood of a point of $L_{X}[9$, p. 83], but it may happen that for some strictly increasing sequence $n(k)$ of positive integers, $\left(S_{n(k)}\right)$ converges uniformly on such a neighborhood. When this happens we shall say that $\left(S_{n(k)}\right)$ overconverges on the neighborhood. (Such overconvergence does occur. For instance, if $\left(O_{n}\right)$ is a sequence of mutually disjoint, simply connected regions such that $O_{1}$ contains $E_{x}$ but not $\mathrm{Cl}\left(E_{x}\right)$ for some $x>1$, then there exists a function $f$, analytic in each $O_{n}$, with modulus of analyticity $x$ with respect to $E$, and a simple polynomial basis $P$, effective on $E$, such that a subsequence $\left(S_{n(k)}\right)$ of $\left(S_{n}\right)$ converges to $f$ uniformly on every compact subset of every $O_{n}$, but does not converge uniformly on any region properly containing some $O_{n}$. See Bourion $[1, \mathrm{pp}$. 268-270] for the proof in the special case $E_{x}=$ the unit disk, $P_{n}(z)=z^{n}$. The proof can be generalized, with $P=$ any interpolation basis belonging to $E$.)

Let $f$ be analytic on $E$ with modulus of analyticity $X$, and let $P$ be any simple polynomial basis effective on $E$. Let $Y$ be some positive number $<1$ and let $n(k)$ be a strictly increasing sequence of positive integers. Let $H$ be the set of all integers $j$ such that $Y n(k) \leqq j \leqq n(k)$ for some $k$. If $\sum_{j \in H} A_{j} P_{j}$ converges compact-openly in $E_{x}$ for some $x>X$, we shall say that $A \cdot P$ is of lacunary structure with respect to $n(k)$, since $A \cdot P$ is then the sum of a series with gaps and a series with a larger modulus of analyticity $\operatorname{than} f$.

THEOREM 5. Let $f$ be analytic on $E$ with modulus of analyticity $X$, and let $P$ be effective on $E$. If the sequence $\left(S_{n}\right)$ of partial sums of $(1.1)$ has a subsequence $\left(S_{n(k)}\right)$ which overconverges on some neighborhood of a point of $L_{x}$, then (1.1) is of lacunary structure with respect to $n(k)$.

Proof. Let $Q$ be any interpolation basis for $E$ and let $G$ be defined by (3.1). For fixed $n, S_{n}=\sum_{j=0}^{n} A_{j} P_{j}=\sum_{h=0}^{n} b_{h n} Q_{h}$ where, by (2.1),

$$
b_{h n}=1 /(2 \pi i) \int_{L_{y}}\left(S_{n}(z) / Q_{n+1}(z)\right) d z
$$


for any $y>1$. Choose $y$ such that $y>X$ and the distance from $L_{y}$ to $E$ is $>1$. Since $Q=G \cdot P$ and $P$ is a basis,

$$
A_{j}=1 /(2 \pi i) \sum_{n=j}^{n} G_{h j} \int_{L_{y}}\left(S_{n}(z) / Q_{n+1}(z)\right) d z
$$

for all $j \leqq n$. Thus if $J$ is the length of $L_{y}$ divided by $2 \pi$, then

$$
\left|A_{j}\right| \leqq \frac{J\left(\max \left|S_{n}(z)\right|, z \text { on } L_{y}\right)}{\left(\min \left|Q_{j+1}(z)\right|, z \text { on } L_{y}\right)} \sum_{n=j}^{n}\left|G_{h j}\right|
$$

for all $j \leqq n$, since $\left|Q_{j+1}(z)\right| \leqq\left|Q_{k+1}(z)\right|$ for all $j \leqq k$ and all $z$ on $L_{y}$. As was pointed out immediately before Theorem 4 , we may assume that every $P_{j}$ is monic. Since $\left(S_{n(k)}\right)$ overconverges, we have

$$
\limsup _{k}\left(\max \left|S_{n(k)}(z)\right|, z \text { on } L_{y}\right)^{1 / n(k)}<y / X
$$

[7, p. 201] and [9, pp. 67 and 77]. Choose $Y$ such that $0<Y<1$ and

$$
\limsup _{k}\left(\max \left|S_{n(k)}(z)\right|, z \text { on } L_{y}\right)^{1 / Y n(k)}<y / X .
$$

Let $H$ be the set of all integers $j$ such that $Y n(k) \leqq j \leqq n(k)$ for some $k$. We first examine the case $K=1$. Let $M(1)$ and $R(1)$ be defined by (3.2) and (3.3). Now $R_{h}(1) \geqq\left|G_{h j}\right| M_{j}(1)$ for all $h$ and $j$, so (in the following six summations let $k$ be the smallest integer such that $j \leqq n(k))$

$$
\limsup _{j \in H}\left(\sum_{h=j}^{n(k)}\left|G_{h j}\right|\right)^{1 / j} \leqq \limsup _{j \in H}\left(\sum_{h=j}^{n(k)} R_{h}(1) / M_{j}(1)\right)^{1 / j} .
$$

From Theorem 4, $\lim \sup _{j \in H}\left(M_{j}(1)\right)^{-1 / j}=1$ so

$$
\limsup _{j \in H}\left(\sum_{n=j}^{n(k)}\left|G_{h j}\right|\right)^{1 / j} \leqq \limsup _{j \in H}\left(\left(\sum_{h=j}^{n(k)} R_{h}(1)\right)^{1 / n(k)}\right)^{n(k) / j} .
$$

But $\lim \sup \left(R_{h}(1)\right)^{1 / h}=1$ by Theorem 1, so

$$
\limsup _{j \in H}\left(\sum_{h=j}^{n(k)} R_{h}(1)\right)^{1 / n(k)}=1,
$$

and since $1 \leqq n(k) / j \leqq 1 / Y$, we get

$$
\limsup _{j \in H}\left(\sum_{h=j}^{n(k)}\left|G_{h j}\right|\right)^{1 / j} \leqq 1 .
$$

Combining this result with (5.1), (5.2), (5.3) and (3.4) we get lim $\sup _{j \in H}\left|A_{j}\right|^{1 / j}<1 / X$. Then by Theorem $4, \sum_{j \in H} A_{j} P_{j}$ converges compact-openly in $E_{x}$ where $x$ $=1 /$ lim $\sup _{j \in H}\left(\left|A_{j}\right|\right)^{1 / j}>X$. This completes the proof of the theorem for the case $K=1$. The proof for the general case follows from a consideration of $\left(P_{j}(K z)\right)$ on $(1 / K) E$. This completes the proof of the theorem. See [6, p. 185] for the theorem in the case $E=$ the closed unit disk, $P_{n}(z)=z^{n}$. 
The converse theorem, for regular points of $f$, was also proved by Ostrowski for the case $E=$ the closed unit disk, $P_{n}(z)=z^{n}$ [5]. This converse theorem was generalized to include not only series, but also sequences of lacunary structure converging to functions analytic on coregular sets, by I. Mosesson in his unpublished Harvard thesis [4]. The following converse to Theorem 5 is therefore a special case of Mosesson's generalization. It also follows immediately from more general work of Walsh.

THEOREM 6. Let $f$ be analytic on $E$ with modulus of analyticity $X$. Let $P$ be effective on $E$ and suppose (1.1) is of lacunary structure with respect to $n(k)$. Then $\left(S_{n(k)}\right)$ converges uniformly in a neighborhood of every regular point of $f$ on $L_{X}$.

Proof. Let $Y$ be a positive number $<1$ such that $\sum_{j \in H} A_{j} P_{j}$ converges compactopenly in $E_{x}$ for some $x>X$, where $H$ is the set of all integers $j$ such that $\operatorname{Yn}(k)$ $\leqq j \leqq n(k)$ for some $k$. For each $k$, let $h(k)$ be the greatest integer $<Y n(k)$. Let $Z_{0}$ be any regular point of $f$ on $L_{X}$. We may assume $A_{j}=0$ for all $j$ in $H$. Thus $S_{h(k)}=S_{n(k)}$ for all $k$. Let $O$ be any compact continuum, not a single point, containing $Z_{0}$ and contained in the complement of $E_{X}$, and let $x=(\max \exp (F(z)), z$ in $O)$. Since $\left(S_{n}\right)$ converges maximally on $E$,

[8, p. 607]. Thus

$$
\lim \sup \left(\max \left|S_{n}(z)\right|, z \text { in } O\right)^{1 / n} \leqq x / X
$$

$$
\begin{aligned}
\limsup _{k}(\max \mid & \left.S_{n(k)}(z) \mid, z \text { in } O\right)^{1 / n(k)} \\
& =\limsup _{k}\left(\max \left|S_{h(k)}(z)\right|, z \text { in } O\right)^{1 / n(k)} \\
& =\limsup _{k}\left(\left(\max \left|S_{h(k)}(z)\right|, z \text { in } O\right)^{1 / n(k)}\right)^{h(k) / n(k)} \leqq(x / X)^{Y}<x / X .
\end{aligned}
$$

The theorem now follows from [7, p. 201].

Corollary Let $P$ be effective on $E$ and let $f$ be analytic on $E$ with modulus of analyticity $X$. Let $\left(A_{n}\right)$ be defined by (1.1) and let $n(k)$ be a sequence of positive integers such that, for some positive number $Y<1, n(k)<Y n(k+1)$. If $A_{n}=0$ for all $n \neq n(k)$, then $L_{X}$ is the natural boundary of $f$.

Proof. $A \cdot P$ cannot converge uniformly in any neighborhood of a point of $L_{X}$ $([9$, p. 83$]$, see [4]). This completes the proof of the corollary.

See Bourion [2, pp. 6-13] for the definitions and theorems in this section, for the case $E=$ the closed unit disk, $P_{n}(z)=z^{n}$.

6. Examples. Let $E$ be the coregular set of all points on and within a Jordan curve and let $P$ be a simple polynomial basis which is orthonormal with respect to integration over $E$ (or around the boundary of $E$ ), with any positive continuous function $W(z)$ allowed as weight function. If $f$ is any function analytic on $E$ with modulus of analyticity $X$, then $f$ can be expanded in the form (1.1), where

$$
A_{k}=\int W(z) f(z) P_{k}(z)^{\prime} d z
$$


(where $P_{k}(z)^{\prime}$ is the complex conjugate of $P_{k}(z)$ ), the convergence being absolute and compact-open in $E_{X}$ [9, pp. 91-97 and 128-130]. Let $Q$ be any interpolation basis for $E$ and let $G$ be defined by (3.1). If $C_{k}$ is the $k$ th column of $G$ then, by (6.1),

$$
\left|G_{n k}\right| \leqq J(\max W(z), z \text { on } E)\left(\max \left|P_{k}(z)\right|, z \text { on } E\right)\left(\max \left|Q_{n}(z)\right|, z \text { on } E\right) \text {, }
$$

where $J$ is the area of $E$ (or the length of $L_{1}$ ). Thus by (3.4),

$$
N\left(C_{k}\right) \leqq \lim _{n} \sup \left(\max \left|Q_{n}(z)\right|, z \text { on } E\right)^{1 / n}=K
$$

and so, by Theorem 2, $P$ is effective on $E$.

The same proof applies to polynomial bases orthonormal over more general coregular sets, with more general weight functions. Almost exactly the same proof applies to the Faber polynomials belonging to an analytic Jordan curve. See [3, pp. 86-87] for a definition of the Faber polynomials.

The zeros of an effective polynomial basis need not be very well behaved. In fact, if $\left(t_{n}\right)$ is any sequence of complex numbers, and $E$ is any coregular set, then there exists a simple polynomial basis $P$, effective on $E$, such that each $t_{n}$ is a zero of infinitely many of the $P_{k}$. To prove this, let $\left(T_{n}\right)$ be the sequence $\left(t_{1}, t_{1}, t_{2}, t_{1}, t_{2}, t_{3}, \ldots\right)$ and choose a sequence $h(n)$ of positive integers such that $h(n+1) \geqq h(n)+2$ and $\lim \sup _{n}\left|T_{n}\right|^{1 / h(n)} \leqq 1$. Let $Q$ be any interpolation basis belonging to $E$, with $Q_{n}(z)=\left(z-Z_{1}\right) \cdots\left(z-Z_{n}\right)$. For $k=h(n)$ let $P_{k}(z)=\left(z-T_{n}\right) Q_{k-1}(z)$, while for $k \neq h(n)$ let $P_{k}(z)=Q_{k}(z)$. If $G$ is defined by (3.1) then $G_{j j}=1$ for all $j$, and $G_{j k}=0$ for $j \neq k$ except when $j=h(n)$ and $k=h(n)-1$, in which case $G_{j k}=T_{n}-Z_{h(n)}$. It is not hard to see directly from definition (3.3), and (3.4), that $P$ is effective on $E$.

\section{BIBLIOGRAPHY}

1. Georges Bourion, Recherches sur l'ultraconvergence, Ann. Sci. Ecole Norm. Sup. 50 (1933), 245-318.

2. - L'ultraconvergence dans les séries de Taylor, Actualités Sci. Indust. No. 472, Hermann, Paris, 1937, pp. 6-47.

3. G. Faber, Uber Tchebycheffsche Polynome, Crelles J. 150 (1920), 79-106.

4. Z. I. Mosesson, Maximal sequences of polynomials, unpublished thesis, Harvard Univ., Cambridge, Mass., 1937.

5. Alexander Ostrowski, Uber eine Eigenschaft gewisser Potenzreihen mit unendlich vielen verschwindenden Koeffizienten, S.-B. Preuss. Akad. 40 (1921), 557-565.

6. - Über Potenzreihen, die überkonvergente Abschnittsfolgen besitzen, S.-B. Preuss. Akad. 42 (1923), 185-192.

7. J. L. Walsh, Overconvergence, degree of convergence, and zeros of sequences of analytic functions, Duke Math. J. 13 (1946), 195-234.

8. - The analog for maximally convergent polynomials of Jentzsch's theorem, Duke Math. J. 26 (1959), 605-616.

9. - Interpolation and approximation by rational functions in the complex domain, 3rd ed., Amer. Math. Soc. Colloq. Publ. Vol. 20, Amer. Math. Soc., Providence, R. I., 1960.

10. J. M. Whittaker, Sur les séries de base de polynômes quelconques, Collection Borel, Gauthier-Villars, Paris, 1949.

Catholic University of America, WASHINGTON, D.C. 